\title{
CROWD-FLOW SIMULATION USING THE POTENTIAL PATHLINE METHOD
}

\author{
SEONGWON NAM \\ Korea Railroad Research Institute, South Korea
}

\begin{abstract}
A new computational method for crowd-flow dynamics was developed to simulate pedestrian flow. It is a simulation method that uses the concept of potential pathline. The physical domain to be analysed is divided into small numerical cells, and potential values are gradually assigned starting from the exit cell. Based on the potential values, the unit direction vector for each cell can be obtained and the pathline from origin to destination drawn. These pathlines are the basic routes for individual pedestrians to move between the origin and the destination. Then, a pedestrian might change the route according to the cell conditions of each location and moves to the destination. Among the many ergonomic factors, the following four were used as the main parameters to determine the route: walking speed, visual angle, straightness probability, and right-hand traffic. It can also be applied to a three-dimensional interlayer movement model. Using the developed analysis program, the author simulated the pedestrian flow for various sample models.

Keywords: computational method, pedestrian flow, rule-based system, way-finding, potential pathline.
\end{abstract}

\section{INTRODUCTION}

When a pedestrian looks for a path in a certain space, the person finds a path by various environmental factors, physical characteristics and experience. Environmental factors include brightness, colour, landmark, path size, vertical and horizontal patterns, signage, etc., and are mainly external factors [1], [2]. Among the many physical characteristics of pedestrians who determine the route and move, representative factors include walking speed, visual angle, straightness, and walking on the right [3], [4]. Crowd flow is common in subway transit stations, terminals, large complex buildings, and stadiums. Because metropolitan subway transit stations are places where passenger flow is overcrowded, predicting crowd flows is important not only for comfort but also for safety [5]. Passengers in subway transit stations during commute times tend to move according to their experience and physical characteristics of the individual rather than external environmental factors. In this study, the author developed a computational method to simulate the crowd flow using the concept of potential pathline of computational fluid dynamics.

\section{POTENTIAL PATHLINE METHOD}

Crowd-flow dynamics (CFD) is a subject that is often studied in the field of transportation and architectural design / planning, but it is physically similar to particle flow, so there are many similarities between the two phenomena. If individual pedestrians are considered as particles, the movement of particles is the movement of individuals, the volume fraction of particles is the density of population, and the trajectory of particle is similar to the pedestrian's movement between origin and destination. Table 1 compares the similarities between the two phenomena. Many methods have been proposed to analyse the crowd flow, which can be divided into discrete models and continuous models.

Representative discrete models are the discrete element model [6], [7], cellular automata model [8], [9], lattice gas discrete model [10], and agent-based model [11]. On the contrary, continuous models are the social force model [12], [13] and forces model [14]. In the discrete model, individual pedestrians move in units of cells. That is, one cell has an area for one 
Table 1: Similarity between particle flow and pedestrian flow.

\begin{tabular}{|l|c|c|}
\hline Item & Particle flow & Pedestrian flow \\
\hline Carrier & Fluid flow & Unit direction vector \\
\hline Mover & Particle & Pedestrian \\
\hline Density & Volume fraction & Population density \\
\hline Velocity & Particle speed & Walking speed \\
\hline $\begin{array}{l}\text { Governing } \\
\text { equation }\end{array}$ & $\begin{array}{c}\text { The equation of } \\
\text { particle motion }\end{array}$ & $\begin{array}{c}\text { The equation of } \\
\text { pedestrian position }\end{array}$ \\
\hline Decision & None & Thinking \\
\hline
\end{tabular}

person, and the movement of the pedestrian moves in each cell unit. On the other hand, in the continuous model, the movement coordinates at each time are calculated by velocity and time. The crowd-flow simulation method developed in this study can trace the individual trajectory in a bilateral passenger flow as well as a multi-layer flow by the Lagrangian approach. When each pedestrian finds a path, the pathline that follows the potential value of each computational grid is the basic path, and the alternative path is searched and moved according to the real-time situation of each cell. When looking for alternative routes, the main parameters of physical characteristics are walking speed, visual angle, straightness, and walking on the right.

In Fig. 1, the factors written in blue letters are environmental external ones and the factors written in black letters are physical characteristic factors. Because external factors depend on how the environment or building is designed, it is difficult to assess quantitatively. The crowd-flow issues are mainly due to individual physical factors that are colliding or dense. In this study, physical characteristics were quantitatively modelled on the assumption that environmental factors are the same. Particle flow has many similarities with pedestrian flow. FVM (Finite Volume Method) and FEM (Finite Element Method) are typical computational fluid dynamics methods for particle flow. These computational fluid dynamics methods have the following characteristics. The space to be analysed is divided into small calculation grids, and the physical property values in each calculation grid are taken as representative average values. Once the flow properties in each computational grid are determined, the particles in the grid are moved by the equation of motion of the particle.

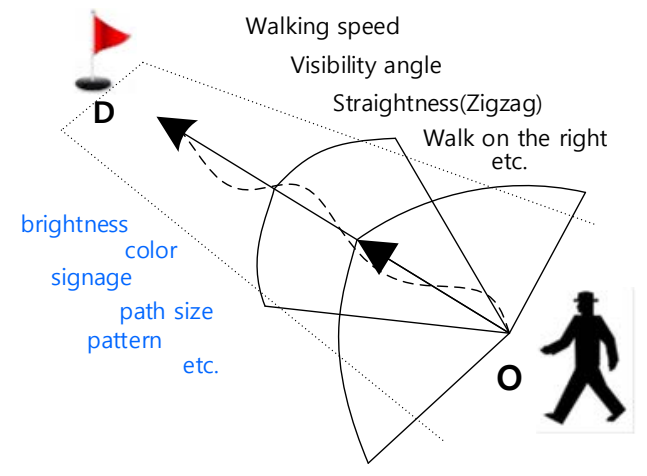

Figure 1: Factors to determine way-finding. 
To illustrate the potential pathline Method developed in this study, the author set up a physical model as shown in Fig. 2. It is a building space with two entrances and one exit, with a wall in the middle. The computational grid and coordinates for the crowd-flow analysis for this model are shown in Fig. 3. The computation domain is divided by the square grid of length $d L$, and the I and $\mathrm{J}$ addresses are given in the $\mathrm{x}$ and $\mathrm{y}$ directions, respectively. It is assumed that the grid size can be adjusted according to the problem, and that only one person can occupy each grid. The average walking speed of people is assumed to be $4 \mathrm{~km} / \mathrm{h}$, and the horizontal and vertical lengths of the grid are $0.5 \mathrm{~m}$ each. The next step is to calculate the potential value for each computational grid. In the potential pathline method, this process is very simple. As a boundary condition, it is sufficient to assign a potential value of 1 to the exit grid. Grids adjacent to the exit grid have a potential value of 2 . That is, if the potential value of grid (I, $\mathrm{J}$ ) is $M$, the potential values of the eight surrounding grids have $M+1$ values. In this way, a potential value map for the entire region can be constructed by sequentially calculating the potential values for the remaining grids. Fig. 4 shows the calculated potential value map.

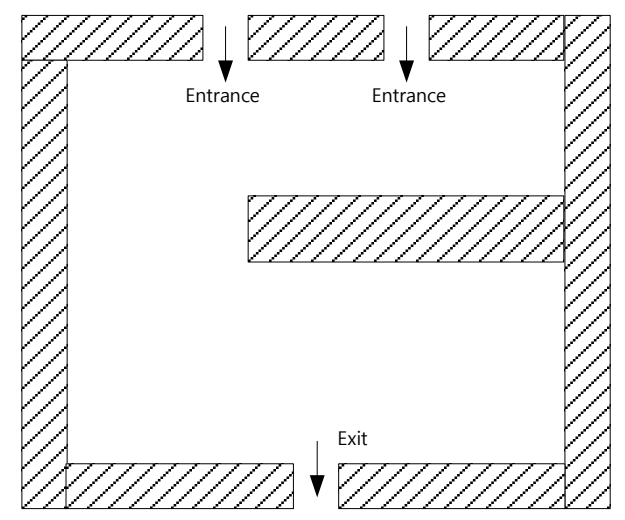

Figure 2: Physical model.

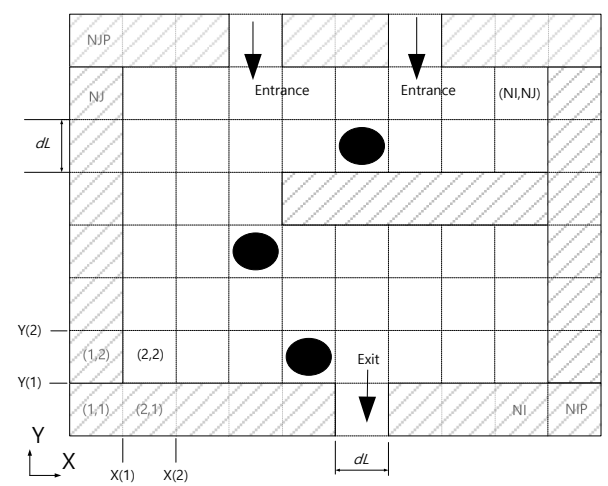

Figure 3: Computational grids and coordinates. 


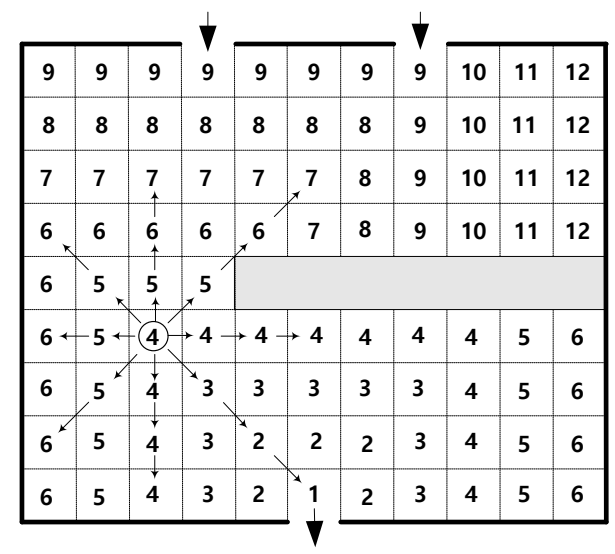

Figure 4: Potential value map.

The next procedure is to calculate the unit direction vector of each grid using a potential value map. In Fig. 4, there are eight grids around the grid with a potential value of 4 . By comparing potential values in each direction, a unit direction vector can be set in the direction having the smallest average value. Potential values in each direction are 4-4-4 in the east, 32-1 in the southeast, 4-4-4 in the south, 5-6 in the southwest, 5-6 in the west, 5-6 in the northwest, 5-6-7 in the north, and 5-6-7 in the northeast. Therefore, it can be seen that the unit direction vector in this grid is southeast. Fig. 5 shows the direction vector set for all the grids and pathline. It is the pathline that connects the lines that follow the direction vector in each grid. If there are no other obstructions, the pedestrians will move along the pathline towards the exit. This can also be deduced from automobile GPS navigation system. When pedestrian set the origin and destination, the route is displayed. Normally, pedestrian will follow the path, but if an obstruction occurs on the path, it will lead to another alternate path. Potential pathline method also finds alternate grid in a similar way. In Fig. 6, let us assume that a person is in grid (I, J) and the direction to move is grid (I, J + 2).

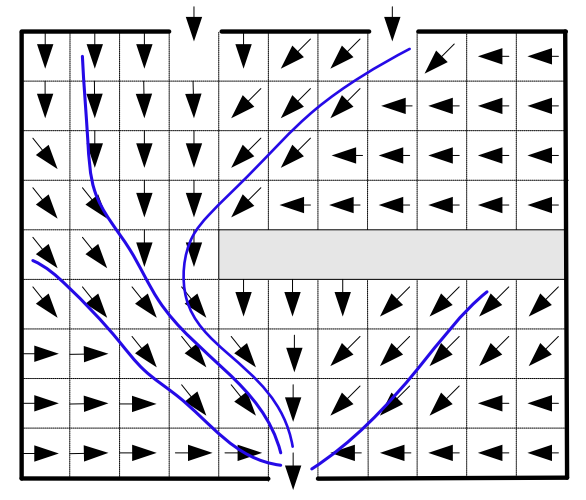

Figure 5: Direction vector and pathline. 


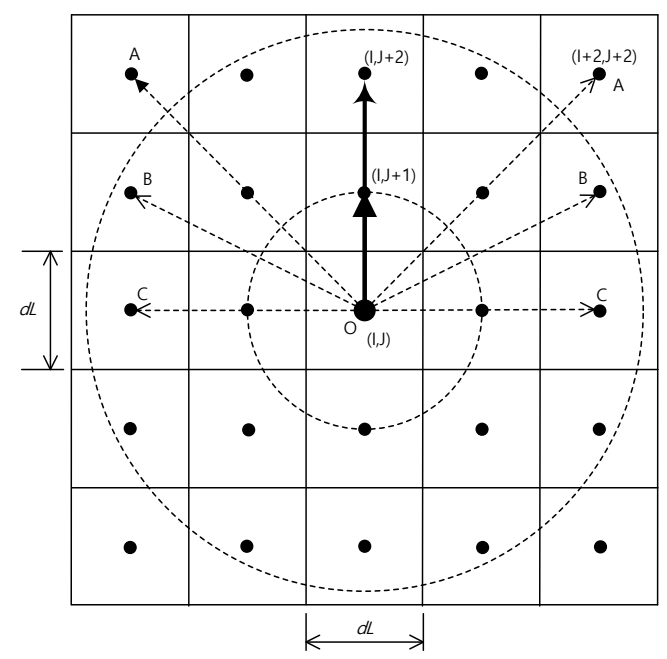

Figure 6: Alternative grid finding.

If the pedestrian is a fast person, it will go to $(I, J+2)$ in the big circle, and if it is slow, it will move to (I, $\mathrm{J}+1$ ) in the small circle. If there are other pedestrians or obstacles already in the grid in the direction he wants to proceed, he will find an alternate grid among the adjacent grids. If he is a person with narrow visibility angle, he will find an alternate grid within the range of angle AOA. If he is a person with wide visibility angle, he will find an alternate grid within the range of angle COC. Generally, pedestrians do not walk straight on a given route. According to ergonomics, pedestrians often walk in zigzags. In this case, he moves to a grid that deviates slightly from the specified direction. In addition, whether he is walking on right side when he encounters an opposite pedestrian or not also affects the route selection. The equation of the pedestrian's position considering the above-mentioned factors is as follows:

$$
\begin{gathered}
\overline{\boldsymbol{S}}_{i j}^{t+d t}=\left\{x_{i j}^{t}+\alpha V_{0} \cos \left(\theta_{1} \pm \theta_{2} \pm \frac{\theta_{3}}{\beta}\right) \Delta t\right\} \overline{\boldsymbol{\imath}} \\
+\left\{y_{i j}^{t}+\alpha V_{0} \sin \left(\theta_{1} \pm \theta_{2} \pm \frac{\theta_{3}}{\beta}\right) \Delta t\right\} \overline{\boldsymbol{J}}
\end{gathered}
$$

where $\overline{\boldsymbol{S}}_{i j}^{t+d t}$ is a new position vector at time $t+d t, x_{i j}^{t}$ and $y_{i j}^{t}$ are positions in the $\mathrm{x}$ and $\mathrm{y}$ directions at time t, $V_{0}$ is walking speed, $\theta_{1}$ is the tangential angle of the pathline at (i, j), $\theta_{2}$ is the angle deviating from the straight line according to the straightness probability, $\theta_{3}$ is the visibility angle, $\alpha$ is the pedestrian group number (specifying a slow pedestrian or a fast pedestrian), $\beta$ is the coefficient of right walking. If the pedestrian is straight ahead, $\theta_{2}$ is 0 , and $\theta_{3}$ is 0 if there are no other pedestrians or obstacles in the direction to move. And, if pedestrians are walking on the right side of the road, this coefficient $\beta$ is 2 . Fig. 7 is a flow chart of the algorithm for finding a new grid to move in unit time. The calculation procedure is as follows. 
PI-32 Computational Methods and Experimental Measurements XIX

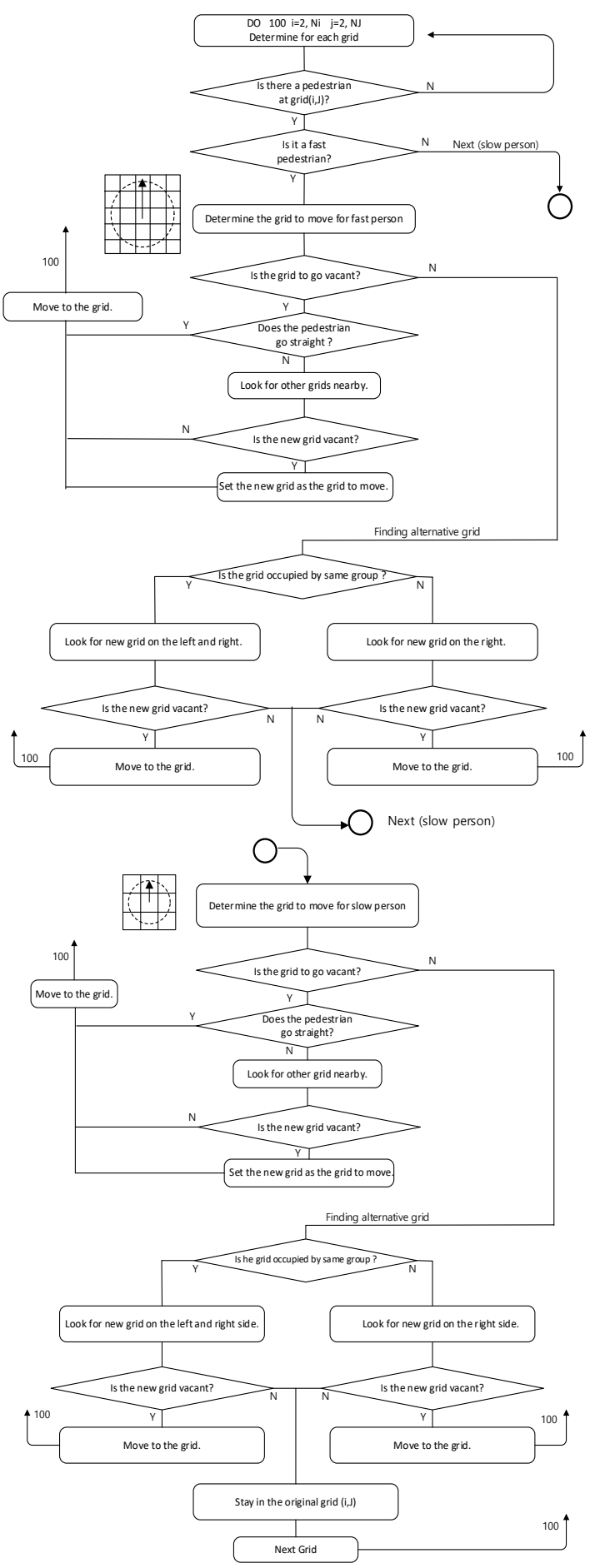

Figure 7: Flow chart to determine the grid to move. 
Walking speed, visibility angle, straightness, and right-hand traffic are assigned to each pedestrian as input data. First, it is decided whether there is a pedestrian in the grid (I, J). If so, decide whether the pedestrian is a fast pedestrian or a slow pedestrian. If he is a fast pedestrian, look for the grid to move to in the big circle, and if it is a slow pedestrian, look for the grid in the small circle. If the new grid on the direction vector side is empty, move to that grid. If the newly found grid is occupied by the pedestrian of the same group, it looks for a new grid to move among the left and right grids, and if it is occupied by the pedestrians of another group, it searches for a new grid to move among grids in the rightward direction. If the newly found grids in the previous routine are not empty, follow the slow pedestrian procedures to find the new grid to move. If the pedestrian can't find the grid to move in these procedures, he will stay in his original position.

\section{RESULTS AND DISCUSSION}

In order to compare the validity and performance of potential pathline method developed in this study, the results using this method were compared with those of other programmes (Fig. 8). The target model is that 20 pedestrians pass through an L-shaped passage of $2.0 \mathrm{~m}$ in width and $20 \mathrm{~m}$ in length. The results of the Exodus show that the pedestrian distribution after 10 seconds is relatively long. The distribution of pedestrians at 10 and 16 seconds is similar, and differs from that at 4 seconds. The results of Pynamics show that, after 10 seconds, when turning left at the curved path, there is a tendency to outward. This is somewhat unrealistic, as pedestrians generally tend to choose the shortest distance. In contrast, these phenomena did not appear in the results using the potential pathline method.

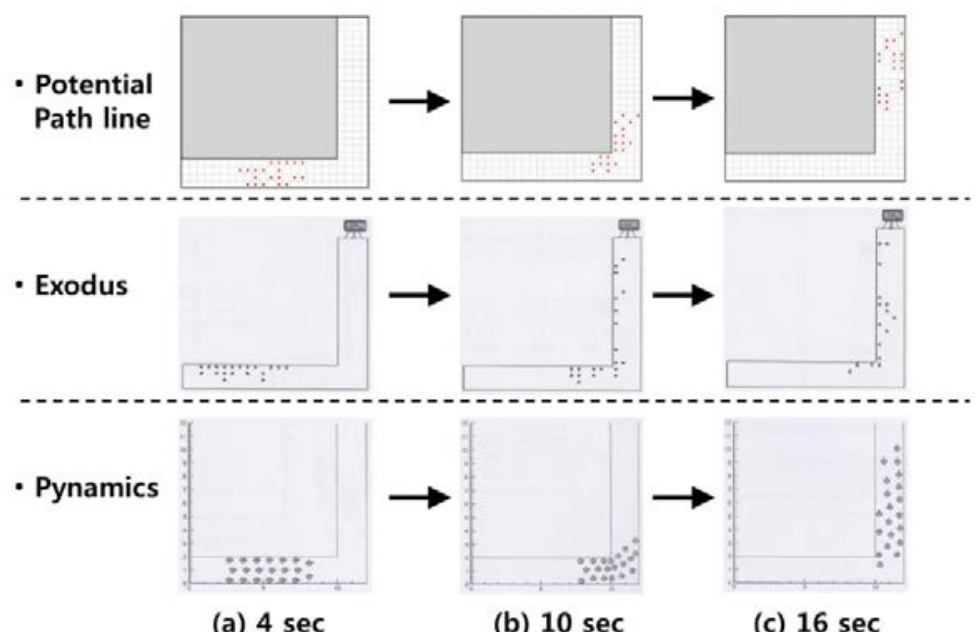

Figure 8: Comparison with other programmes results.

For the next comparison model, the results of Helbing et al.'s study are given as an example [13]. 200 people in a room of $15 \mathrm{~m}$ in width and $15 \mathrm{~m}$ in height move at a speed of $1 \mathrm{~m} / \mathrm{s}$ through $1 \mathrm{~m}$ wide exit on the right, and Fig. 9 shows a snapshot. The crowd-flow characteristics in this model are arching and clogging around the exit. Both analytical results are qualitatively similar. But, the results of Helbing et al.'s research show that the arcs are almost the same radius from the exit, which is somewhat excessive. In contrast, the results of the potential pathline method are much more natural. 


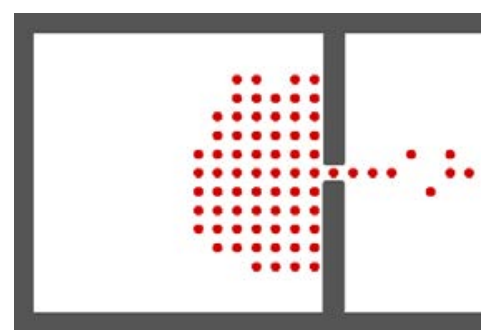

(a)

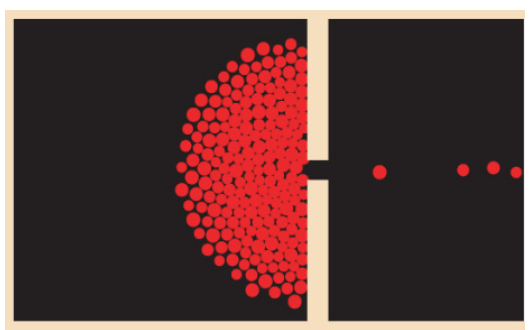

(b)

Figure 9: Comparison with Helbing et al.'s result (snapshot). (a) Potential pathline method; (b) Helbing et al.’s result [13].

Fig. 10 shows a comparison of snapshot of the effect of door location. The author simulated the change of the paths of the audience leaving the theatre after the play. The author compared the cases where the doors are only on the rear side of the theatre and those in each direction. In the case of Fig. 9(a), since the doors are at the rear, the path of the audience in front of the stage is lengthened, and it can be seen that the exit is stagnated. Comparing the figures after 10 seconds, it seems that the audience in the case of Fig. 9(b) exits much more efficiently.

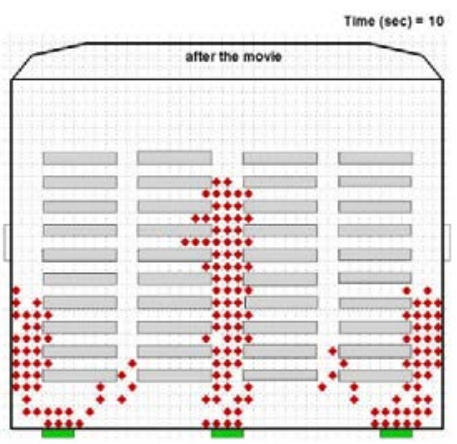

(a)

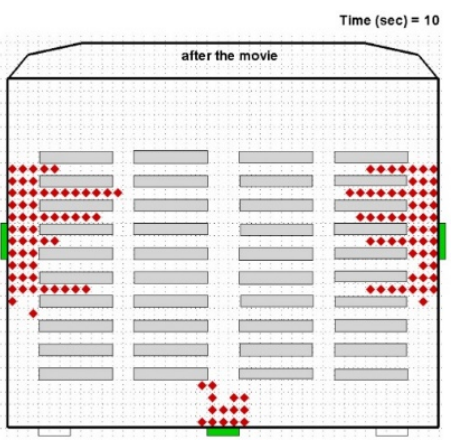

(b)

Figure 10: Comparison of the effect of door location (snapshot). (a) Doors are in the back side; (b) Doors are in each direction.

\section{CONCLUSIONS}

Crowd-flow simulation is often used as a design tool for the structural design of a railway station, terminal, complex multiple building, and stadium. In this study, a new numerical analysis method was developed to simulate pedestrian flow. It is a computational analysis method using the concept of potential pathline. The domain to be analysed is divided into small calculation cells, and a potential value is gradually given starting from the exit cell. Based on the potential values, the direction vector for each cell can be obtained and the pathline following it drawn. These pathlines are the basic routes for individual pedestrians to move between the origin and the destination. In this simulation method, walking speed, visibility angle, straightness, and right-hand traffic were used as main parameters. Using the developed simulation programme for various pedestrian flow cases, the pedestrian flow at 
L-shaped route, evacuation from a room, pedestrian count flow in passage, and exit patterns for the doors' location were simulated.

\section{ACKNOWLEDGEMENT}

This research was supported by a grant (PK1901C4) from the R\&D programme of Korea Railroad Research Institute (KRRI), Republic of Korea.

\section{REFERENCES}

[1] Hughes, R.L., The flow of human crowds. Annual Review of Fluid Mechanics, 35, pp. 169-182, 2003.

[2] Moussaid, M., Helbing, D. \& Theraulaz, G., How simple rules determine Pedestrian Behavior and Crowd Disasters. PNAS, 108(17), pp. 6884-6888, 2011.

[3] Polus, A., Schofer, J.L., \& Ushpiz, A., Pedestrian flow and level of service. J. Transp. Eng. Proc. ASCE, 109, pp. 46-57. 1983.

[4] Nam, S., Development of potential pathline method for railway passenger flow analysis. Journal of the Korean Society for Railway, 21(8), pp. 830-837, 2018.

[5] Daamen, W., Modelling Passenger Flows in Public Transport Facilities. PhD thesis, Delft University of Technology, 2004.

[6] Nam, S., Development of algorithm for passenger flow analysis based on DEM. Journal of the Korean Society for Railway, 8(4), pp. 337-341, 2005.

[7] Nam, S., Analysis of pedestrian flow characteristics in subway station. Journal of the Korean Society for Railway, 9(3), pp. 271-276, 2006.

[8] Nagal, K. \& Schreckenber, M., A cellular automation model for freeway traffic. Journal of Physics, 2(12), pp. 2221-2229, 1992.

[9] Burstedde, C., Klauck, K. \& Schadschneider, A., Simulation of pedestrian dynamics using a two-dimensional cellular automaton. Physica A, 295, pp. 507-525, 2001.

[10] Guo, R. \& Huang, H., A mobile lattice gas model for simulating pedestrian evacuation. Physica A, 387, pp. 580-586, 2008.

[11] Heliovaara, S., Korhonen, T., Hostikka, S. \& Ehtamo, H., Counterflow model for agebt-based simulation of crowd dynamics. Building and Environment, 48, pp. 89100, 2012.

[12] Helbing, D. \& Molnar, P., Social force model for pedestrian dynamics. Physical Review E, 51, pp. 4282-4286, 1995.

[13] Helbing, D., Farkas, I. \& Vicsek, T., Simulating dynamical features of escape panic. Nature, 407(28), pp. 487-490, 2000.

[14] Lohner, R., On the modelling of pedestrian motion. Applied Mathematical Modelling, 34, pp. 366-382, 2010. 